\title{
Hypergraphical Metric Spaces and Fixed Point Theorems
}

\author{
Xiaodong Li $\mathbb{D},{ }^{1}$ Farhan Khan, ${ }^{2}$ Gohar Ali $\mathbb{D}^{3},{ }^{3}$ Lubna Gul, ${ }^{3}$ and Muhammad Sarwar $\mathbb{D}^{2}$ \\ ${ }^{1}$ Huanghe Jiaotong University, Jiaozuo 454950, Henan, China \\ ${ }^{2}$ Department of Mathematics, University of Malakand, Chakdara Dir (L), Khyber Pakhtunkhwa, Pakistan \\ ${ }^{3}$ Department of Mathematics, Islamia College Peshawar, Khyber Pakhtunkhwa, Pakistan
}

Correspondence should be addressed to Xiaodong Li; li-xiaodong-li@outlook.com, Gohar Ali; gohar.ali@icp.edu.pk, and Muhammad Sarwar; sarwarswati@gmail.com

Received 23 March 2021; Accepted 6 June 2021; Published 27 June 2021

Academic Editor: Ali Ahmad

Copyright (c) 2021 Xiaodong Li et al. This is an open access article distributed under the Creative Commons Attribution License, which permits unrestricted use, distribution, and reproduction in any medium, provided the original work is properly cited.

Hypergraph is a generalization of graph in which an edge can join any number of vertices. Hypergraph is used for combinatorial structures which generalize graphs. In this research work, the notion of hypergraphical metric spaces is introduced, which generalizes many existing spaces. Some fixed point theorems are studied in the corresponding spaces. To show the authenticity of the established work, nontrivial examples and applications are also provided.

\section{Introduction}

Graph theory has been used to study the various concepts of navigation in an arbitrary space. A work place can be denoted as a vertex in the language of graph theory, and edges denote the connections between these places (vertices). Hypergraph is a generalization of graph in which an edge can join any number of vertices. Hypergraph is used for combinatorial structures which generalize graphs. The applications of hypergraph can be found in Engineering sciences, many areas of Computer Science, and almost all areas of Mathematics.

Moreover, directed hypergraphs are used in computer science, particularly in the development of data mining, software testing, image segmentation and processing, information security, and communication networks.

\section{Preliminaries}

Frechet et al. initiated the concept of metric spaces in 1906, which open the door for entering into a more waste and new field in the world of mathematics. Upon this foundation, different researchers introduced different generalized metric spaces and studied various fixed point results with applications. In this way, we refer some recent developments in $[1-3]$. About basic notions of graph theory, we refer to the readers [4-6] and references therein.

In 1736, Leonhard Euler put the framework of graph theory by studying the historical problem of seven bridges of Konigsberg and prefigured the concept of topology. Echinique [7] deliberated fixed point theory by using graph. Jachymsky [8] replaced the order structure with a graph structure on a metric space and studied the well-known Banach contraction principle. Aleomraninejad et al. [9] gave the concept of some fixed point results on metric space with a graph, in which they presented some iterative results for G-contractive and G-nonexpansive mappings on graphs. Samreen et al. [10] investigated some fixed point theorems in b-metric space endowed with graph. Argoubi et al. [11] presented some fixed point results and its applications by considering self-mappings defined on a metric space endowed with a finite number of graphs.

Shukhla et al. [12] gave the concept of graphical metric space which is a generalized setting in fixed point theory and established some fixed point results with applications. Abbas et al. [13] presented some fixed point results for set contractions on metric spaces with a directed graph. In 2017, Debanath and Neog [14] initiated the concept of start point 
on a metric space endowed with a directed graph. They offered the alternate concept of start point in a directed graph and provided the characterizations which are necessary for a directed graph having start point. Kumam et al. [15] presented graphic contraction mapping in b-metric space and established some fixed point results with applications.

Motivated by the above results, combining the notion of hypergraph and metric, we introduced hypergraphical metric space which generalized the concept of graphical metric space. In hypergraphical metric space, vertices of graph are replaced by edges. Some conclusions, examples, and an application to integral equation are also presented to authenticate the acceptation and unifying power of obtained generalizations. For iterative numerical schemes, the interesting readers can refer the recent papers $[16,17]$.

\section{Hypergraph and Hypergraphical Metrics}

Definition 1. Hypergraph is real generalization of graph. The edges of hypergraph connect any number of nodes. Formally it is a pair, i.e., $G_{H}=(\zeta, \xi)$ in which $\zeta$ represents set of vertices and $\xi$ is a set of nonempty subsets of $\zeta$ called hyperedges or simply edges.

Definition 2. Hypergraph $G_{H}$ is said to be directed hypergraph if $G_{H}=(V, \xi)$ where $V \neq \varnothing$ is a finite set and is known as the set of nodes of $G_{H}$ and $\xi$ is the set of directed hyperedges, where a hyperedge or hyperarc $e=(T$ $(e), H(e))$ is a directed hyperedge with $|T(e)|>0$ and $|H(e)|>0$ and both are disjoint. $H(e)$ and $T(e)$ represent head and tail, respectively, where hyperedge ends and starts and contains set of nodes.

Definition 3. The size of directed hypergraph $G_{H}$ is defined as the sum of the tail and head nodes of each hyperedge together with the number of nodes of the hypergraph, i.e., $\left|G_{H}\right|=|V|+\sum_{e \in \mathcal{\varepsilon}}(|T(e)|+|H(e)|)$.

Definition 4. A directed path in a directed hypergraph is a sequence of nodes and hyperedges such that each edge points from a node in the sequence to its successor in the sequence.

Let $G_{H}=(v, \xi)$ be a directed hypergraph and $\left(v_{i}, v_{j} \in v\right)$ is a directed path from $s$ to $t$ in $G_{H}$, which represent the sequence $\left(\pi_{s, t}\right)$ of the form $\pi_{s \rightarrow t}=\left(v_{1}, e_{1}, v_{2}, e_{2}, \ldots\right.$, $\left.e_{n-1}, v_{n}\right): n>0$ such that $v_{i} \in v, \forall i \in\{1,2,3, \ldots, n\}$ and $e_{j} \in \xi, \forall j \in\{1,2,3, \ldots, n-1\} . \quad v_{1} \in s \Rightarrow v_{1} \in T\left(e_{1}\right) \quad$ and $v_{n}=t \Longrightarrow v_{n} \in H\left(e_{i-1}\right) \cap T\left(e_{i}\right), \forall i \in\{2, \ldots, n-1\}$.

Definition 5. The edges which connect other edges are called hyperdelta edges; that is, vertices of these edges are also edges and denoted by $\Delta$.

Definition 6. A hypergraph in which we assign numerical value, i.e., nonnegative real numbers $[0 \infty)$ to their edges is called labeled graph.
Definition 7 (see [12]). Let $\zeta \neq \varnothing$ set endowed with graph $G_{m}$ and $d_{G_{m}}: \zeta * \zeta \longrightarrow R$ be a function satisfying the following condition:

$$
\begin{aligned}
& \left(\mathrm{GM}_{1}\right) \cdot d_{G_{m}}(a, b)=0, \text { if } a=b \\
& \left(\mathrm{GM}_{2}\right) \cdot d_{G_{m}}(a, b)>0, \text { if } a \neq b \\
& \left(\mathrm{GM}_{3}\right) \cdot d_{G_{m}}(a, b)=d_{G_{m}}(b, a), \forall a, b \in \zeta \\
& \left(\mathrm{GM}_{4}\right) \cdot(a p b)_{G_{m}}, \\
& c \in(a p b)_{G_{m}} \text { implies } d_{G_{m}}(a, b) \leq d_{G_{m}}(a, c)+ \\
& d_{G_{m}}(c, b), \forall a, b, c \in \zeta
\end{aligned}
$$

Then, the mapping $d_{G_{m}}$ is called a graphical metric on $\zeta$, and the pair $\left(\zeta, d_{G_{m}}\right)$ is called graphical metric space.

By combining the concept of hypergraph and graphical metric space, we introduced the following notion of hypergraphical metric spaces.

Definition 8. Suppose $\zeta$ be a nonempty set endowed with hypergraph $G_{H}$ such that $V\left(G_{H}\right)=\zeta$ and let $\xi$ represent hyperedges of $G_{H}$ such that each hyperedge $e$ represents nonempty subset of $\zeta$. Suppose the mapping $d_{G_{H}}: \xi * \xi \longrightarrow R$ satisfying the following condition:

$$
\begin{aligned}
& \left(\mathrm{HGM}_{1}\right) \cdot d_{G_{H}}\left(e_{i}, e_{j}\right)=0 \text {, if } e_{i}=e_{j} \\
& \left(\mathrm{HGM}_{2}\right) \cdot d_{G_{H}}\left(e_{i}, e_{j}\right)>0 \text {, if } e_{i} \neq e_{j} \\
& \left(\mathrm{HGM}_{3}\right) \cdot d_{G_{H}}\left(e_{i}, e_{j}\right)=d_{G_{H}}\left(e_{j}, e_{i}\right), \forall e_{i}, e_{j} \in \xi \\
& \left(\mathrm{HGM}_{4}\right) \cdot\left(e_{i} p e_{j}\right)_{G_{H}} \\
& e_{k} \in\left(e_{i} p e_{j}\right)_{G_{H}} \operatorname{implies}_{G_{H}}\left(e_{i}, e_{j}\right) \leq d_{G_{H}}\left(e_{i}, e_{k}\right)+ \\
& d_{G_{H}}\left(e_{k}, e_{j}\right), \forall e_{i}, e_{j}, e_{k} \in \xi
\end{aligned}
$$

Then, $d_{G_{H}}$ is called a hypergraphical metric on $\xi$, and $\left(\xi, d_{G_{H}}\right)$ is said to be hypergraphical metric space.

Remark 1. We noted that hypergraphical metric space is the real generalization of graphical metric space; that is, every graphical metric space is hypergraphical metric but converse is not true.

Example 1. Let $\zeta=\left\{v_{1}, v_{2}, v_{3}, v_{4}, v_{5}, v_{6}\right\}$ be the set of vertices, and let $\xi=\left\{\left\{v_{1}\right\},\left\{v_{2}\right\},\left\{v_{3}\right\},\left\{v_{4}, v_{5}, v_{6}\right\}\right\}$ which is composed by edges of hypergraph $G_{H}$. Now, let us define a function $d_{G_{H}}: \xi * \xi \longrightarrow R^{+}$by

$$
d_{G_{H}}\left(e_{i}, e_{j}\right)= \begin{cases}0, & \text { if } e_{i}=e_{j}, \\ 5 A, & \text { if } e_{i}, e_{j} \in\left\{\left\{v_{1}\right\},\left\{v_{2}\right\}\right\} e_{i} \neq e_{j}, \\ 3 A, & \text { if } e_{i}, e_{j} \in\left\{\left\{v_{1}\right\},\left\{v_{3}\right\}\right\} e_{i} \neq e_{j}, \\ A, & \text { if } e_{i}, e_{j} \in\left\{\left\{v_{2}\right\},\left\{v_{3}\right\}\right\} e_{i} \neq e_{j}, \\ 4 A, & \text { if } e_{i}, e_{j} \in\left\{\left\{v_{1}\right\},\left\{v_{4}, v_{5}, v_{6}\right\}\right\} e_{i} \neq e_{j}, \\ 6 A, & \text { if } e_{i}, e_{j} \in\left\{\left\{v_{2}\right\},\left\{v_{4}, v_{5}, v_{6}\right\}\right\} e_{i} \neq e_{j}, \\ 2 A, & \text { if } e_{i}, e_{j} \in\left\{\left\{v_{4}, v_{5}, v_{6}\right\},\left\{v_{2}\right\}\right\} e_{i} \neq e_{j},\end{cases}
$$

where $A>1$ is the positive real number. Evidently $d_{G_{H}}$ is not a graphical metric because

$$
d_{G_{H}}\left(\left\{v_{1}\right\},\left\{v_{2}\right\}\right) \nless d_{G_{H}}\left(\left\{v_{1}\right\},\left\{v_{3}\right\}\right)+d_{G_{H}}\left(\left\{v_{3}\right\},\left\{v_{2}\right\}\right),
$$


since $5 A>3 A+A$.

On the other hand,

$$
d_{G_{H}}\left(\left\{v_{1}\right\},\left\{v_{2}\right\}\right) \leq d_{G_{H}}\left(\left\{v_{1}\right\},\left\{v_{4}, v_{5}, v_{6}\right\}\right)+d_{G_{H}}\left(\left\{v_{4}, v_{5}, v_{6}\right\},\left\{v_{2}\right\}\right) \text {. }
$$

In this case, we have $5 A \leq 4 A+6 A$. Therefore, $d_{G_{H}}$ is the hypergraphical metric space.

Not every hypergraphical metric space is metric. Let us provide an example as follows.

Example 2. Let $X=[0,1]$; here, $X$ interval means the weight of edges of $G_{H}$, where $G_{H}$ be the hypergraph such that its edges can be defined as $\xi_{G_{H}}=\Delta U\left\{e_{i}\right.$, $\left.e_{j}: e_{i}, e_{j} \in(1,1) e_{i} \leq e_{j} \wedge i, j \in N\right\}$. Define a mapping $d_{G_{H}}: \xi * \xi \longrightarrow R^{+}$by

$$
d_{G_{H}}\left(e_{i}, e_{j}\right)= \begin{cases}0, & \text { if } e_{i}=e_{j} ; \\ e_{i} * e_{j}, & \text { if } e_{i}, e_{j} \in(0,1] e_{i} \neq e_{j} ; \\ e_{i}+e_{j}, & \text { otherwise. }\end{cases}
$$

Then, $d_{G_{H}}$ is a hypergraphical metric on $\xi$ and $\left(\xi, d_{G_{H}}\right)$ is a hypergraphical metric space obviously where $d_{G_{H}}$ not a metric on $\xi$.

Definition 9. Let $\left(\xi, d_{G_{H}}\right)$ be hypergraphical metric space. An open ball $B_{G_{H}}(e, \varepsilon)$ with center $e$ and radius $\epsilon$ is defined as

$$
B_{G_{H}}(e, \varepsilon)=\left\{e^{\prime}:\left(e p e^{\prime}\right)_{G_{H}}, d_{G_{H}}\left(e, e^{\prime}\right)<\varepsilon\right\} .
$$

Since $\xi\left(G_{H}\right) \sqsupseteq \Delta$, therefore, we have $e \in B_{G_{H}}(e, \varepsilon)$. Hence, $B_{G_{H}}(e, \varepsilon)$ is nonempty $\forall e \in \xi$ and $\varepsilon>0$. The collection

$$
B=\left\{B_{G_{H}}(e, \varepsilon): e \in \xi, \varepsilon>0\right\},
$$

which is the neighborhood system for the topology $T_{G_{H}}$ on $\xi$ induced by the hypergraphical metric $d_{G_{H}}$. A subset $S$ of $\xi$ is called open if for every $e \in S$ there exist an $\varepsilon>0$ such that $B_{G_{H}}(e, \varepsilon) \subset S$; of course, a subset $T$ of $\xi$ is called closed if its complement $T^{c}$ is open.

\section{Lemma 1. Every open ball in $\xi$ is an open set.}

Proof. Let $e^{\prime} \in B_{G_{H}}(e, \varepsilon)$ for some $e \in \xi$ and $\varepsilon>0$. Let $\alpha=$ $\varepsilon-d_{G_{H}}\left(e, e^{\prime}\right)>0$ and $e^{\prime \prime} \in B_{G_{H}}\left(e^{\prime}, \alpha\right)$; by definition, we have $\left(e p e^{\prime}\right)_{G_{H}}$ and $\left(e^{\prime} p e^{\prime \prime}\right)_{G_{H}}$ and so that $\left(e p e^{\prime \prime}\right)_{G_{H}}$. Now, from Property (4) of hypergraphical metric space, $d_{G_{H}}\left(e^{\prime \prime}, e\right) \leq d_{G_{H}}\left(e^{\prime \prime}, e^{\prime}\right)+d_{G_{H}}\left(e^{\prime}, e\right)<\alpha+d_{G_{H}}\left(e^{\prime}, e\right)=\varepsilon-$ $d_{G_{H}}\left(e^{\prime}, e\right)+d_{G_{H}}\left(e^{\prime}, e\right)=\varepsilon$. Hence, $B_{G_{H}}\left(e^{\prime}, \alpha\right) \subset B_{G_{H}}(e, \varepsilon)$. Hence, every open ball in $\xi$ is an open set.

Definition 10. Suppose $\left(\xi, d_{G_{H}}\right)$ is hypergraphical metric space and $\left\{e_{n}\right\}$ be a sequence in $\xi$, then $\left\{e_{n}\right\}$ is called convergent and converges to $e \in \xi$ if for given $\varepsilon>0$ there $\exists n_{\mathrm{o}} \in N$ such that $d_{G_{H}}\left(e_{n}, e\right) \leq \varepsilon, \forall n>n_{\mathrm{o}}$. Obviously the sequence $\left\{e_{n}\right\}$ is convergent and converges to $e$ if and only if $\lim _{n \longrightarrow \infty} d_{G_{H}}\left(e_{n}, e\right)=0$.
Remark 2. . The limit of a sequence in hypergraphical metric space may not be unique as clear from the following example.

Example 3. let $X$ be the set of vertices of hypergraph, and we take $\xi\left(G_{H}\right)$ to be the set of subsets of $X$ such that each subset represents an edge of the hypergraph $G_{H}$. Now, we labeled some edges from the set $2_{A} \bigcup\{0\}$, where $2_{A}=\left\{1 / 2^{n}: n \in \mathbb{N}\right\}$. We define $\xi\left(G_{H}\right)=\left\{e_{i}, e_{j}: e_{i} \leq e_{j}, i, j \in \mathbb{N}\right\}$. Define a mapping $d_{G_{H}}: \xi \times \xi \longrightarrow \mathbb{R}^{+}$by

$$
d_{G_{H}}\left(e_{i}, e_{j}\right)= \begin{cases}0, & \text { if } e_{i}=e_{j} ; \\ e_{i} \times e_{j}, & \text { if } e_{i}, e_{j} \in 2_{A} e_{i} \neq e_{j} ; \\ \frac{1}{2}, & \text { otherwise. }\end{cases}
$$

Clearly, $d_{G_{H}}$ is a hypergraphical metric on $\xi$. Now, let us consider the sequence $\left\{e_{n}\right\}$ in $\xi$ where $e_{n}=1 / 2^{n}, \forall n \in \mathbb{N}$; then, for any fixed $k \in \mathbb{N}$, we have

$$
d_{G_{H}}\left(\frac{1}{2^{n}}, \frac{1}{2^{k}}\right)=\frac{1}{2^{n+k}} \longrightarrow 0, \quad n \longrightarrow \infty .
$$

Therefore, the sequence $\left\{1 / 2^{n}\right\}$ converges to $1 / 2^{k}$ for every fixed $k \in \mathbb{N}$.

Lemma 2. Let $\left(\xi, d_{G_{H}}\right)$ be a hypergraphical metric space with induced hypergraphical topology $T_{G_{H}}$. Then, $T_{G_{H}}$ is $T_{1}$ but not generally Hausdorff, i.e., $T_{2}$.

Proof. We want to show that for every $e \in \xi$, the singleton set $\{e\}$ is a closed subset of $\xi$ or the set $\xi-\{e\}$ is an open subset of $\xi$. For this, let us suppose $e^{\prime} \in \xi-\{e\}$, then clearly $e^{\prime} \neq e$ and $d_{G_{H}}\left(e, e^{\prime}\right)>0$. Now, let us take $d_{G_{H}}\left(e, e^{\prime}\right)=2 \varepsilon>0 \longrightarrow(*)$. Then, clearly $e$ does not belong to $B_{G_{H}}\left(e^{\prime}, \varepsilon\right)$. Suppose on contrary that $e \in B_{G_{H}}\left(e^{\prime}, \varepsilon\right)$, then $d_{G_{H}}\left(e, e^{\prime}\right)<\varepsilon$ which is contradiction to $(*)$. Hence, $B_{G_{H}}\left(e^{\prime}, \varepsilon\right) \subset \xi-\{e\}$ is open, and hence hypergraphical metric space is not Hausdorff.

Remark 3. Let $\left(\xi, d_{G_{H}}\right)$ be hypergraphical metric space in previous remark, then $1 / 2$ is limit point of the sequence $e_{n}=\left\{1 / 2^{n}\right\} \in \xi$, but for any $k \in \mathbb{N}$, if $k>1$, we have $\lim _{n \rightarrow \infty} d_{G_{H}}\left(1 / 2^{n}, 1 / 2^{k}\right)=0 \neq d_{G_{H}}\left(1 / 2,1 / 2^{k}\right)$. Therefore, a hypergraphical metric does not need to be continuous.

Definition 11. Let $\left(\xi, d_{G_{H}}\right)$ be hypergraphical metric space, and $\left\{e_{n}\right\} \in \xi$ is a sequence. Then, $\left\{e_{n}\right\}$ is called Cauchy if for given $\varepsilon>0$ there exist $n_{\text {。 }}$ belong to $\mathbb{N}$ such that $d_{G_{H}}\left(e_{n}, e_{m}\right)<\varepsilon, \forall n, m>n_{\circ}$; obviously the sequence $\left\{e_{n}\right\}$ is Cauchy sequence $\Leftrightarrow \lim _{n \longrightarrow \infty} d_{G_{H}}\left(e_{n}, e_{m}\right)=0$.

Definition 12. A hypergraphical metric space $\left(\xi, d_{G_{H}}\right)$ is called complete if each Cauchy sequence in $\xi$ converges in $\xi$. Suppose $G_{H}^{\prime}$ is another hypergraph such that each $e \in \xi\left(G_{H}^{\prime}\right)$ is subset of $V\left(G_{H}^{\prime}\right)$, that is, $e \subseteq V\left(G_{H}^{\prime}\right)$, then $\left(\xi, d_{G_{H}}\right)$ is called 
$G_{H}^{\prime}$-complete if every $G_{H}^{\prime}$ termwise connected Cauchy sequence in $\xi$ converges in $\xi$.

In this paper, we suppose that hypergraph $G_{H}$ is considered to be directed. We include directed path $(\mathrm{p})$ between edges and denote by $[e]_{G_{H}}^{l}=\left\{e^{\prime} \in \xi\right.$ : directed path from to of length $l\}$.

\section{Main Results}

In this section, we provide fixed point results in hypergraphical metric space; for this, we need various definitions to support our main results.

Definition 13. Suppose $\left(\xi, d_{G_{H}}\right)$ is hypergraphical metric space and $F: \xi \longrightarrow \xi$ is a mapping and $G_{H}^{*}$ is subhypergraph of $G_{H}$ such that $\xi\left(G_{H}^{*}\right) \supseteq \Delta$. Then, $F$ is said $\left(G_{H}, G_{H}^{*}\right)$-hypergraphical contraction on $\xi$ if the conditions given below are satisfied.

$G_{H C 1}: F$ preserves edges in $G_{H}^{*}$ such that $e \in \xi\left(G_{H}^{*}\right) \Rightarrow F_{e} \in \xi\left(G_{H}^{*}\right)$

$G_{H C 2}$ : there exists $\alpha \in[0,1)$, such that for $e_{i} e_{j} \in \xi\left(G_{H}^{*}\right)$ and $F_{e_{i}}, F_{e_{j}} \in \xi\left(G_{H}^{*}\right), d_{G_{H}}\left(F_{e_{i}}, F_{e_{j}}\right) \leq \alpha d_{G_{H}}\left(e_{i}, e_{j}\right)$ for all $e_{i} e_{j} \in \xi\left(G_{H}^{*}\right)$

Here, we assign the hypergraphical distance between the edges of $G_{H}^{*}$, and hypergraphical contraction decreases the distance by factor $\alpha \in[0,1)$. The sequence $\left\{e_{n}\right\}$ having earliest value $e_{0} \in \xi$ is called $F$-picard sequence if $e_{n}=F_{e_{n-1}}, \forall n \in \mathbb{N}$. Further, we suppose that $G_{H}^{*}$ is a subhypergraph of $G_{H}$ such that $\xi\left(G_{H}^{*}\right) \supseteq \Delta$. The next theorem is the dominant outcome which gives sufficient conditions for the convergence of picard sequence yielded by $\left(G_{H}, G_{H}^{\prime}\right)$-hypergraphical contraction on $G_{H}^{\prime}$-complete hypergraphical metric space.

Theorem 1. Suppose $\left(\xi, d_{G_{H}}\right)$ is $G_{H}^{\prime}$-complete hypergraphical metric space and $F: \xi \longrightarrow \xi$ be a $\left(G_{H}, G_{H}^{\prime}\right)$-hypergraphical contraction and also satisfies the following conditions. (1) There exist $e_{0} \in \xi$ such that $F_{e_{0}} \in\left[e_{0}\right]_{G_{H}^{\prime}}^{l}$, for some $l \in \mathbb{N}$. (2) If $G_{H}^{\prime}$-termwise connected F-picard sequence $\left\{e_{n}\right\}$ converges in $\xi$, then a limit $e^{\prime} \in \xi$ of $\left\{e_{n}\right\}$ exists and $n_{0} \in \mathbb{N}$, such that $\left(e_{n}, e^{\prime}\right) \in \xi\left(G_{H}^{\prime}\right), \forall n>n_{0}$.

Then, there exist $e^{*} \in \xi$ such that the $F$-picard sequence $\left\{e_{n}\right\}$ of initial value $e_{0}$ is $G_{H}^{\prime}$-termwise connected and converges to $e^{*}$ and $\mathrm{Fe}^{*}$.

Proof. Suppose $e_{0} \in \xi$ such that $F_{e_{0}} \in\left[e_{0}\right]_{G_{H}^{\prime}}^{l}$ for some $l \in \mathbb{N}$ and $\left\{e_{n}\right\}$ is $F$-picard sequence having initial value $e_{0}$, then $\left[\left\{e_{i}^{\prime}\right\}\right]_{i=0}^{l}$ is a path such that $e_{0}=e_{0}^{\prime}, F e_{0}=e_{l}^{\prime}$, and $\left(e_{i-1}^{\prime}, e_{i}^{\prime}\right) \in \xi\left(G_{H}^{\prime}\right)$ for $i=1,2,3, \ldots, l$. As $F$ is a $\left(G_{H}, G_{H}^{\prime}\right)$-hypergraphical contraction, we have

$$
\left(F e_{i-1}^{\prime}, F e_{i}^{\prime}\right) \in \xi\left(G_{H}^{\prime}\right), \quad \text { for } i=1,2,3, \ldots, l .
$$

Therefore, $\left[\left\{T e_{i}^{\prime}\right\}\right]_{i=0}^{l}$ represent a path from $F e_{0}^{\prime}=F e_{o}=$ $e_{1}$ to $T e_{l}^{\prime}=F^{2} e_{0}=e_{2}$ of length $l$ and so $e_{2} \in\left[e_{1}\right]_{G_{H}^{\prime}}^{l}$; proceeding similarly, we get the path $\left[\left\{T^{n} e_{i}^{\prime}\right\}\right]_{i=0}^{l}$ from $F^{n} e_{0}^{\prime}=$
$F^{n} e_{0}=e_{n}$ to $F^{n} e_{l}^{\prime}=F^{n} F e_{0}=e_{n+1}$ of length $l$. Hence, $e_{n+1} \in\left[e_{n}\right]_{G_{H}^{\prime}}^{l}, \forall n \in \mathbb{N}$; thus, $\left\{e_{n}\right\}$ is a $G_{H^{\prime}}^{\prime}$-termwise connected sequence. Since $\left(F^{n} e_{i-1}^{\prime}, F^{n} e_{i}^{\prime}\right) \in \xi\left(G_{H}^{\prime}\right)$ for $i=1,2,3, \ldots, l$ and $n \in \mathbb{N}$. Using condition $\left(G_{H} C_{2}\right)$, we have

$$
d_{G_{H}}\left(F^{n} e_{i-1}^{\prime}, F^{n} e_{i}^{\prime}\right) \leq \alpha^{n}\left(d_{G_{H}}\left(e_{i-1}^{\prime}, e_{i}^{\prime}\right)\right) .
$$

Since $G_{H}^{\prime}$ is a subgraph of $G_{H},\left\{e_{n}\right\}$ and is a termwise connected sequence in $G_{H}^{\prime}$, by using (10), the following relation holds $\forall n \in \mathbb{N}, m>n$ :

$$
d_{G_{H}}\left(e_{n}, e_{n+1}\right)=d_{G_{H}}\left(F^{n} e_{0}, F^{n+1} e_{0}\right) \leq \sum_{i=1}^{l} \alpha^{n} d_{G_{H}}\left(e_{i-1}^{\prime}, e_{i}^{\prime}\right)=\alpha^{n} F_{l} .
$$

where $F_{l}=\sum_{i=1}^{l} d_{G_{H}}\left(e_{i-1}{ }^{\prime}, \dot{e}_{i}\right)$. Again as the sequence $\left\{e_{n}\right\}$ is $G_{H}^{\prime}$-termwise connected, therefore, $n, m \in \mathbb{N}$ with $m>n$, we have

$$
\begin{aligned}
d_{G_{H}}\left(e_{n}, e_{m}\right) & \leq \sum_{i=n}^{m-1} d_{G_{H}}\left(e_{i}, e_{i+1}\right) \leq \sum_{i=n}^{m-1} \alpha^{i} F_{l}=\sum_{i=n}^{m-1} \alpha^{i-n+n} F_{l} \\
& =\alpha^{n}\left[\sum_{i=n}^{m-1} \alpha^{i-n} F_{l}\right]=\frac{\alpha^{n}}{1-\alpha} .
\end{aligned}
$$

Since $\alpha \in(0,1]$, we obtain $\lim _{n, m \rightarrow \infty} d_{G_{H}}\left(e_{n}, e_{m}\right)=0$. Therefore, $\left\{e_{n}\right\}$ is a Cauchy sequence in $\xi$. From $G_{H}^{\prime}$-completeness of $\xi$, the sequence $\left\{e_{n}\right\}$ converges in $\xi$. And from condition (2), there exist $e^{*} \in \xi$ and $n_{0} \in N$, such that $\left(e_{n}, e^{*}\right) \in \xi\left(G_{H}^{\prime}\right), \forall n>n_{0} \quad$ and $\quad \lim _{n \longrightarrow \infty} d_{G_{H}}\left(e_{n}, e^{*}\right)=0$. Thus, the sequence $\left\{e_{n}\right\}$ converges to $e^{*} \in \xi$. Now, if $\left(e_{n}, e^{*}\right) \in \xi\left(G_{H}^{\prime}\right)$ for all $n>n_{0}$ by using $\left(G_{H} C_{2}\right)$, we obtain

$$
d_{G_{H}}\left(e_{n+1}, F e^{*}\right)=d_{G_{H}}\left(F e_{n}, F e^{*}\right) \leq \alpha d_{G_{H}}\left(e_{n}, e^{*}\right), \quad \text { for all } n>n_{0},
$$

since $\lim _{n \longrightarrow \infty} d_{G_{H}}\left(e_{n}, e^{*}\right)=0$.

Therefore,

$$
\lim _{n \longrightarrow \infty} d_{G_{H}}\left(e_{n+1}, F e^{*}\right)=0 .
$$

A similar result holds if $\left(e^{*}, e_{n}\right) \in \xi\left(G_{H}^{\prime}\right)$, and hence the sequence $\left\{e_{n}\right\}$ converges to both $e^{*}$ and $F e^{*}$.

If we replace $\xi$ by the set of vertices instead of edges, we get the following corollary.

Corollary 1. Suppose $\left(\xi, d_{G_{H}}\right)$ is $G_{H}^{\prime}$ - complete graphical metric space and $F: \xi \longrightarrow \xi$ be a $\left(G_{H}, G_{H}^{\prime}\right)$-graphical contraction and also satisfies the following conditions.

(1) There exist $x_{0} \in \xi$ such that $F_{x_{0}} \in\left[x_{0}\right]_{G_{H}^{\prime}}^{l}$. For some $l \in N$.

(2) If $G_{H}^{\prime}$-termwise connected, F-picard sequence $\left\{x_{n}\right\}$ converges in $\xi$. Then, a limit $x^{\prime} \in \xi$ of $\left\{x_{n}\right\}$ exists and $n_{0} \in N$, such that $\left(x_{n}, x^{\prime}\right) \in \xi\left(G_{H}^{\prime}\right), \forall n>n_{0}$.

Then, there exist $x^{*} \in \xi$ such that the $F$-picard sequence $\left\{e_{n}\right\}$ of earliest value $x_{0}$ is $G_{H}^{\prime}$-termwise connected and converges to $x^{*}$ and $F x^{*}$. 


\section{Remark 4}

Corollary 1 is the result of Shukla [12].

Remark 5. Theorem 1 confirms only convergent of a picard sequence yielded from a $\left(G_{H}, G_{H}^{\prime}\right)$-hypergraphical contraction on a $G_{H}^{\prime}$-complete hypergraphical metric space. Next example displays that no one should appreciate this theorem as an existence theorem in $G_{H}^{\prime}$-complete hypergraphical metric space.

Example 4. Suppose $\zeta$ be the nonempty set of vertices of hypergraph $G_{H}$ and $\xi\left(G_{H}\right)$ be the set of subset of $\zeta$ such that each subset represents an edge of the hypergraph $G_{H}$. Note (here, $G_{H}$ means weighted hypergraph) that we labeled some edges of $G_{H}$ from set $2_{A} \cup\{0\}$. Here, $2_{A}$ is the set, that is, $2_{A}=\left\{1 / 2^{n}: n \in N\right\}$ and $\xi\left(G_{H}\right)=\left\{e_{i}, e_{j}: e_{i} \leq e_{j}\right.$ and $\left.i, j \in N\right\}$. Define a mapping $d_{G_{H}}: \xi^{*} \xi \longrightarrow \mathbf{R}^{+}$by

$$
d_{G_{H}}\left(e_{i}, e_{j}\right)= \begin{cases}0, & \text { if } e_{i}=e_{j} ; \\ e_{i} * e_{j}, & \text { if } e_{i}, e_{j} \in 2_{A} e_{i} \neq e_{j} ; \\ \frac{1}{2}, & \text { otherwise. }\end{cases}
$$

Then, $d_{G_{H}}$ is hypergraphical metric on $\xi$ and $\left(\xi, d_{G_{H}}\right)$ is $G_{H}$-complete hypergraphical metric space; now, here we define a mapping.

$\mathrm{F}: \xi \longrightarrow \xi$ by

$$
F e= \begin{cases}\frac{e}{2}, & \text { if } e \in 2_{A} ; \\ \frac{1}{2}, & \text { if } e=0\end{cases}
$$

It should be noted that $F$ is hypergraphical contraction having $\alpha=1 / 4, \forall e \in 2_{A}$, and we have $(e, F e) \in \xi\left(G_{H}\right)$, which implies that $F e \in[e]_{G_{H}^{\prime}}^{l}$. Also any $G_{H^{-}}$-termwise connected and convergent sequence in $\xi$ is constant or monotonic decreasing subsequence with respect to usual order of the sequence $\left\{1 / 2^{n}\right\}$ and having at least one limit $e^{\prime}$ such that property (2) of contraction theorem hold surely. However, there is no fixed point in $\xi$ of $F$. As mentioned, that convergent sequence's limit may not be unique in hypergraphical metric space $G_{H}$. Therefore, we provide one more definition that is as follows.

Definition 14. Suppose $\left(\xi, d_{G_{H}}\right)$ is hypergraphical metric space and $F: \xi \longrightarrow \xi$ is a mapping, then property $(P)$ holds for the quadruple $\left(x, d_{G_{H}}, G_{H}^{\prime}, T\right)$, that is:

$(P)$ : whenever a $G_{H}^{\prime}$-termwise connected, F-picard sequence $\left\{x_{n}\right\}$ having limits $e_{i}$ and $e_{j}$ where $e_{i} \in \xi$ and $e_{j} \in F(\xi)$, then $e_{i}=e_{j}$.
We represent all fixed point of a set by $\mathrm{Fix} * F$, and notation for this is $\xi_{F}=\left\{e \in \xi:(e, F e) \in \xi\left(G_{H}^{\prime}\right)\right\}$.

Remark 6. If we chose $\xi\left(G_{H}\right)=\xi^{*} \xi$, then it is clear to check that quadruple $\left(\xi, d_{G_{H}}, G_{H}^{\prime}, F\right)$ satisfies property $(P)$ for arbitrary subhypergraph $G_{H}^{\prime}$.

Example 5. Let $X, G_{H}$, and $d_{G_{H}}$ be those which is used in Example 1. And $\xi\left(G_{H}^{\prime}\right)=\Delta \cup\left\{e_{i}, e_{j}: e_{i}, e_{j} \in(0,1), e_{i} \leq\right.$ $\left.e_{j} \wedge i, j \in N\right\}$.

$$
F e= \begin{cases}e, & \text { if } \in Q \cap[0,1] \\ 1, & \text { otherwise; }\end{cases}
$$

Then, the quadruple $\left(\xi, d_{G_{H}}, G_{H}^{\prime}, F\right)$ has the property $(P)$. In the next theorem, we want to give enough condition for the existence of fixed point of a $\left(G_{H}, G_{H}^{\prime}\right)$-graphical contraction.

Theorem 2. Suppose $\left(\xi, d_{G_{H}}\right)$ is $G_{H}^{\prime}-$ complete hypergraphical metric space and $F: \xi \longrightarrow \xi$ is $\left(G_{H}, G_{H}^{\prime}\right)$-hypergraphical contraction, it holds the following: (1) there exist $e_{0} \in \xi$ such that $T_{e_{0}} \in\left[e_{0}\right]_{G_{H}^{\prime}}^{l}$ for some $l \in N$; (2) if a $G_{H}^{\prime}$-termwise connected $T$-picard sequence $\left\{e_{n}\right\}$ converges in $\xi$, then there $\exists e^{\prime} \in \xi$ of $\left\{e_{n}\right\}$ which is limit point and $n_{0} \in N$ such that $\left(e_{n}, e^{\prime}\right) \in \xi\left(G_{H}^{\prime}\right)$ for all $n>n_{0}$; then, there exist $e^{*} \in \xi$ such that the T-picard sequence $\left\{e_{n}\right\}$ having earliest value $e_{0}$ is $G_{H}^{\prime}$-termwise connected and converges to $e^{*}$ and Te*. Also, if the quadruple $\left(\xi, d_{G_{H}}, G_{H}^{\prime}, F\right)$ satisfies property $(P)$, then there must be fixed point of $F$ in $\xi$.

Proof. From Theorem 3.2, F-picard sequence $\left\{e_{n}\right\}$ having earliest value $e_{0}$ converges to $e^{*}$ and $T e^{*}$. As $e^{*} \in \xi$ and $\mathrm{Fe}^{*} \in F(\xi)$, therefore, by property $(P)$, it is essential that $T e^{*}=e^{*}$. Hence, $\mathrm{F}$ has fixed point $e^{*}$ which is a fixed point of $T$.

Remark 7. In the above result, fixed point of $\mathrm{F}$ exists due to property $(P)$; it is very important to note that in Example 5, every condition of above result holds except property $(P)$. However, Fix $(F)=\Phi$. Therefore, in the above theorem property, $(P)$ remains unused.

\section{Applications}

Let $I>0$ and $\zeta=C([0,1], R)$ represent set of weights of edges which is in the form of real continuous function on weighted interval $[0, I]$. We give a special application of fixed point theory for examining integral equations of $\zeta$; we show that according to certain condition, the actuality of a lower or upper solution of an integral equation ensures the solution of integral equation. Let $B_{H}=\{e \in X: 0<$ $\inf _{p \in[0, I]} e(p)$ and $\left.e(p) \leq 1, t \in[0, I]\right\}$. Here, we have $G_{H}=$ $G_{H}^{\prime} \quad$ and $\xi\left(G_{H}\right)=\Delta\left\{e_{i}, e_{j}\left\{e_{i}, e_{j} \in B_{H} e_{i}(p) \leq e_{j}(p) \forall p\right.\right.$ 
$\in[0, I]\}$. Consider that hypergraphical metric space which is given below, that is, $d_{G_{H}}: \xi * \xi \longrightarrow R$ is given by

$$
d_{G_{H}}\left(e_{i} e_{j}\right)= \begin{cases}0, & \text { if } e_{i}=e_{j}, \\ \sup _{p \in[0, I]}\left\{\ln \left(\frac{1}{e_{i}(p) \cdot e_{j}(p)}\right)\right\}, & \text { if } e_{i}, e_{j} \in B_{H} e_{i} \neq e_{j}, \\ 1, & \text { otherwise. }\end{cases}
$$

So, $\left(\xi, d_{G_{H}}\right)$ is $G_{H}^{\prime}$-complete hypergraphical metric space. Here, we suppose following integral equation:

$$
e_{i}(p)=\int_{0}^{I} g(p, q) h\left(q, e_{i}(q)\right) \mathrm{d} q,
$$

where $g:[0, I] *[0, I] \longrightarrow[0,+\infty)$ and $h:[0, I] * R \longrightarrow R$ are continuous functions. Mapping $\beta_{I} \in C([0, I], R)$ is called lower solution of (19) if $\beta(p) \leq \int_{0}^{I} g(p, q) h(q, \beta(q)) \mathrm{d} q$, $p \in[0, I]$. Here, we want to prove that the existence of lower solution of (Z1) conforms the existence of solution of (19). Let us suppose that the operator $F: \xi \longrightarrow \xi$ is defined by

$$
F e_{i}(p)=\int_{0}^{I} g(p, q) h\left(q, e_{i}(q)\right) \mathrm{d} q,
$$

and sufficient conditions are provided for existence of fixed point of (20) in $\xi$, and obviously that fixed point is solution of (19).

Theorem 3. Consider that coming conditions hold the following:

(a) $\ldots h(q, *): R \longrightarrow R$ is increasing function on $(0,1]$ for every $q \in[0, I]$. Moreover, $\inf _{p \in[0, I]} g(p, q)>0$ and $g(p, q) h(q, 1) \leq I^{-1}$.

(b) There exist $\alpha \in(0,1)$ and $\rho \in[1,+\infty)$ such that for $e_{i}$, $e_{j} \in \xi$ and $\left(e_{i}, e_{j}\right) \in \xi, \forall q, r \in[0, I]$.

$$
\begin{gathered}
h(q, e(q)) h\left(r, e_{j}(r)\right) \geq\left[e_{i}(q) e_{j}(r)\right]^{\alpha}, \\
\int_{0}^{I} \int_{0}^{I} g(p, q) g(p, r) \mathrm{d} q \mathrm{~d} r \geq \rho, \quad \forall p \in[0, I] .
\end{gathered}
$$

Then, existence of a lower solution of (19) in $B_{H}$ confirms the existence of solution of (19) in $\xi$.

Proof. By condition (b), for $e_{i}, e_{j} \in \xi$ also $\left(e_{i}, e_{j}\right) \in \xi$, and $P \in[0, I]$, we derived

$$
\begin{aligned}
& \ln \left(\frac{1}{F\left(e_{i}\right)(p) \cdot F\left(e_{j}\right)(p)}\right) \\
& \quad=\ln \left(\frac{1}{\int_{0}^{I} \int_{0}^{I} g(p, q) g(p, r) h\left(q, e_{i}(q)\right) h\left(r, e_{j}(r)\right) \mathrm{d} q \mathrm{~d} r}\right) \\
& \quad \leq \ln \left(\frac{1}{\inf p_{p \in[0, I]}\left[e_{i}(p) e_{j}(p)\right]^{\alpha} \int_{0}^{I} \int_{0}^{I} g(p, q) g(p, r) \mathrm{d} q \mathrm{~d} r}\right) \\
& \quad=\ln \left(\frac{1}{F\left(e_{i}\right)(p) \cdot F\left(e_{j}\right)(p)}\right) \\
& \quad=\ln \left(\frac{1}{\int_{0}^{I} \int_{0}^{I} g(p, q) g(p, r) h\left(q, e_{i}(q)\right) h\left(r, e_{j}(r)\right) \mathrm{d} q \mathrm{~d} r}\right) \\
& \quad \leq \ln \left(\frac{1}{\int_{0}^{I} \int_{0}^{I} g(p, q) g(p, r)\left[e_{i}(q) e_{j}(r)\right]^{\alpha} \mathrm{d} q \mathrm{~d} r}\right) \\
& \quad \leq \ln \left(\frac{1}{\inf _{p \in[0, I]}\left[e_{i}(p) e_{j}(p)\right]^{\alpha} \int_{0}^{I} \int_{0}^{I} g(p, q) g(p, r) \mathrm{d} q \mathrm{~d} r}\right) \\
& \quad \leq \ln \left(\frac{1}{\rho \inf f_{p \in[0, I]}\left[e_{i}(p) e_{j}(p)\right]^{\alpha}}\right) \\
& \quad \alpha d_{G_{H}}\left(e_{i}(p), e_{j}(p)\right) z z .
\end{aligned}
$$

Then, we have

$$
\begin{aligned}
d_{G_{H}}\left(F e_{i}, F e_{j}\right) & =\sup _{p \in[0, I]} \ln \left(\frac{1}{F\left(e_{i}\right)(p) F\left(e_{j}\right)(p)}\right) \\
& \leq \alpha d_{G_{H}}\left(e_{i}(P), e_{j}(P)\right) .
\end{aligned}
$$

Further, for $e_{i}, e_{j} \in B_{H}$, and $e_{i}(p) \leq e_{j}(p), \forall p \in[0, I]$ and from condition (a) we have $\inf _{p \in[0, I]} F\left(e_{i}\right)(p)>0$ and

$$
\begin{aligned}
F\left(e_{i}\right)(p) & =\int_{0}^{I} g(p, q) h\left(q, e_{i}(q)\right) \mathrm{d} q \\
& \leq \int_{0}^{I} g(p, q) h(1,1) \mathrm{d} q \leq 1, \\
F\left(e_{1}\right)(p) & =\int_{0}^{I} g(p, q) f\left(q, e_{i}(q)\right) \mathrm{d} q \\
& \leq \int_{0}^{I} g(p, q) h\left(q, e_{j}(q)\right) \mathrm{d} q=F\left(e_{j}\right)(p) .
\end{aligned}
$$


Consequently, the existence of lower solution of equation (19), i.e., $\beta \in B_{H}$ implies that property of Theorem 3 holds. Also, the quadruple $\left(\xi, d_{G_{H}}, G_{H}^{\prime}, F\right)$ has property $(p)$. Hence, all conditions of Theorem 2.8 are satisfied. Thus, the operator $\mathrm{F}$ has a fixed point which is solution of integral equation (19) in $\xi$.

\section{Data Availability}

The data used to support this research are included within the paper.

\section{Conflicts of Interest}

The authors declare that they have no conflicts of interest.

\section{Authors' Contributions}

X. Li analyzed the results and finalized the paper. G. Ali supervised the work. L. Gul proved the main results. F. Khan wrote the first draft of the paper. M. Sarwar verified the results.

\section{References}

[1] I. Altun and H. Simsek, "Some fixed point theorems on ordered metric spaces and application," Fixed Point Theory and Applications, vol. 2010, no. 1, 11 pages, Article ID 621469, 2010.

[2] W. Sintunavarat, "Fixed point results in b-metric spaces approach to the existence of a solution for nonlinear integral equations," Serie A. Matemáticas, vol. 110, no. 2, pp. 585-600, 2016.

[3] E. Karapinar and M. Noorwali, "Dragomir and Gosa type inequalities on b-metric spaces," Journal of Inequalities and applications, vol. 29, 2019.

[4] H. M. Nagesh and V. R. Girish, "On the entire Zagreb indices of the line graph and line cut-vertex graph of the subdivision graph," Open Journal of Mathematical Sciences, vol. 4, no. 1, pp. 470-475, 2020.

[5] M. Numan, S. I. Butt, and A. Taimur, "Super cyclic antimagic covering for some families of graphs," Open Journal of Mathematical Sciences, vol. 5, no. 1, 2021.

[6] F. Asif, Z. Zahid, and S. Zafar, "Leap Zagreb and leap hyperZagreb indices of Jahangir and Jahangir derived graphs," Engineering and Applied Science Letter, vol. 3, no. 2, p. 18, 2020.

[7] F. echenique, "A short and constructive proof of Tarski's fixed-point theorem," International Journal of Game Theory, vol. 33, no. 2, pp. 215-218, 2005.

[8] J. Jacek, "The contraction principle for mappings on a metric with a graph," Proceeding of the American Mathematical Society, vol. 136, no. 4, pp. 1359-1373, 2008.

[9] S. Aleomraninejad, S. Rezapour, and N. Shahzad, "Some fixed point results on a metric space with a graph," Topology and its Applications, vol. 159, no. 3, 2012.

[10] M. Samreen, T. Kamran, and N. Shahzad, "Some fixed point theorems in b-Metric space endowed with graph," Abstract and Applied Analysis, vol. 2013, Article ID 967132, 9 pages, 2013.

[11] H. Argoubi, B. Samet, M. Turinici, B. Samet, and M. Turinici, "Fixed Point results on a metric space endowed with a finite number of graphs and applications," Czechoslovak Mathematical Journal, vol. 64, no. 1, pp. 241-250, 2014.

[12] S. Shukla, "Graphical metric space: a generalized setting in fixed point theory," Serie A, Matematicas, vol. 111, no. 3, 2016.

[13] M. Abbas, M. R. Alfuraidan, A. R. Khan, and T. Nazir, "Fixed point results for set-contractions on metric spaces with a directed graph," Fixed Point Theory and Applications, vol. 2015, no. $1,2015$.

[14] M. Neog and P. Debnath, "Fixed points of set valued mappings in terms of start point on a metric space endowed with a directed graph," Mathematics, vol. 5, 2017.

[15] N. Chuensupantharat, P. Kumam, V. Chauhan, and D. Singh, "Graphic contraction mapping via graphical b-metric spaces with applications," Article in the Bulletin of the Malaysian Society Series, vol. 2, 2018.

[16] S. Regmi, I. K. Argyros, and S. George, "Convergence analysis for a fast class of multi-step Chebyshev-Halley-type methods under weak conditions," Open Journal of Mathematical Sciences, vol. 4, no. 1, pp. 34-43, 2021.

[17] S. Regmi, C. Argyros, C. Argyros, I. K. Argyros, and S. George, "On some iterative methods with frozen derivatives for solving equations," Open Journal of Mathematical Sciences, vol. 5, no. 1, pp. 209-217, 2021. 